\title{
System Identification and Modelling of Front Wheel Drive Electric Wheelchairs
}

\author{
Xiaoqi Chen*, J Geoff Chase*, Patrick Wolm*, Isaac Anstis*, John Oldridge*, William Hanbury-Webber*, \\ Rodney Elliot*, Warren Pettigrew** \\ *Dept of Mechanical Engineering, University of Canterbury, \\ Private bag 4800, Christchurch 8140, New Zealand \\ Tel: 643364 2987; email:xiaoqi.chen@canterbury.ac.nz \\ **Dynamic Controls Ltd, 17 Print Place, Middleton, \\ Christchurch 8015, New Zealand email: wpettigrew@dynamiccontrols.com
}

\begin{abstract}
A wireless data acquisition and control platform has been developed to model electric powered wheelchairs. The test-bed integrates sensors, embedded controller, and the motorised mechanical system. Real time data acquisition and analysis is performed in dSpace. The motion control is executed via RS232 Bluetooth and Radio-Control receiver. Test procedures have been designed to find parameters of the wheelchairs, including centre of gravity, moment of inertia, and electric constants. These parameters can then be used in dynamics modeling. Both kinematics model and force model of the wheelchair have been established, using Simulink as a platform to derive the model. The developed experimental setup not only facilitates system identification and modelling of motorised wheelchairs, and wheeled mobile robots in general; but also serves as an excellent case study for mechatronics and control design.
\end{abstract}

\section{INTRODUCTION}

Future electric powered wheelchairs should meet the needs of a large user base of aged and disabled people. Intensive research efforts have been put in to develop highly effective home-installed devices which will provide the user with continuous assistance in achieving high mobility without compromising comfort. During the 1998-2025 period, the world's elderly population (ages 65 and above) is predicted to more than double (Bureau of the Census, 1999). In UK, the number of wheelchair users is about $1.5 \%$ total population, and about $72 \%$ users are over 60 (NHS, 1996)

Market forecasts for power wheelchair, scooter, and all terrain mobility systems indicate strong growth based on new technology and demand from people wanting improved mobility. The markets at US\$1.7 billion in 2005 are expected to grow to US\$5.3 billion by 2012 (WinterGreen, 2004). Growing obese population drives demand for bariatric mobility equipment. The number of obese people is increasing at an alarming rate. In 2004, there were 68.9 million obese persons in North America and this number was expected to increase to 74.7 million in 2006 (Frost \& Sullivan, 2004). Bariatric mobility products are projected to be the fastest growing mobility aid segment in the near future. In response to the increasing market needs of mobility systems which are safe and easy to drive, intensive research effort has devoted to accurate dynamics modelling and robust control.

The transportation needs of individuals with disabilities were surveyed (Linden et al., 1996). Modern designs demand that the user is less engaged in the control process, and the interaction between the device and human-friendly. In other word, the devices should possess a high level of intelligence in their controls, actions, and interactions with the user, offering him/her a high level of comfort and functionality. A mixed control mode was considered as a better alternative for human-machine interactions for users with great movement disability. (Stefanov et al., 2000).

The posture of wheelchair users experiencing external perturbations was examined through the use of a tilt platform (Kamper et al., 1999). The work revealed that spinal cord injury subjects lost balance at perturbation levels seen during normal braking makeovers. Powered four-wheeled wheelchairs with two driving wheels and two small sized front wheels were studied (Boiadzjiev et al., 2002). The work proposed a controller with a special module that analyses user's commands before their execution.

The problem of controlling the movements of a handicapped person's motorised wheelchair was investigated from a practical point of view (Boquete et al., 1999). Low level PID controller directly controls the drivers of the chair's motors, with a classic PID (proportional-integral-derivative) control loop. The high level control was implemented by means of neural techniques to ensure linear and angular speeds of the wheelchair follow those indicated by a trajectory generator. The influence of caster orientations and driving speeds on the reverse control of electric-powered wheelchairs was examined (Ding et al., 2004).

In order to prototype wheelchair control functions, manmachine interface, and predict the performance of wheelchair, a virtual model of the wheelchair dynamics need to be created. This model can be further validated quickly and repeatedly, without the difficulties of physically setting the 
wheelchair up for each test. This requires a robust wireless test-bed which transmits sensor data and receives motor control instructions without data corruption and timing issues.

This work presents experimental work on instrumentation and parameterisation of motorised wheelchairs. It firstly discusses instrumentation setup, sensors used and motion control system. The wireless data acquisition and control platform has been developed. The test-bed integrates sensors, the motion controller with a PWM drive interface, and the motorised mechanical system. Experimental procedures have been designed to find parameters of the wheelchairs, including centre of gravity, moment of inertia, and electric constants. Finally the kinematics and force models of the wheelchairs are discussed.

\section{CONTROL AND INSTRUMENTS}

\subsection{Wireless Control}

Fig. 1 shows the top level view of the wireless test-bed of the wheelchair control prototyping. Key components of the integrated mechatronics system include hardware and software platform, wireless communication, sensors and motor control.

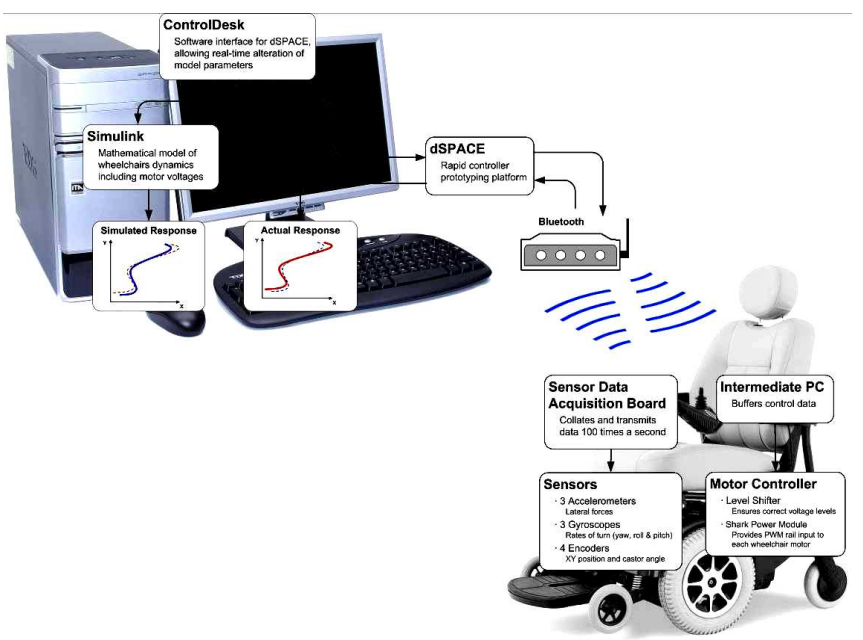

Fig. 1. Wheelchair instrumentation system overview.

dSPACE and ControlDesk: The hardware and software package is the cornerstone of the whole wireless platform. It is through this system that real time data acquisition and analysis is performed. ControlDesk also enables the parameters, such as the input motor voltages, to be varied in real time.

Simulink: Simulink, a simulation program within Matlab, was used to build the computer model of the wheelchair. It was interfaced closely with ControlDesk for testing.

Bluetooth: Bluetooth was employed for wireless communications between the wheelchair and base PC. The wireless data link enabled the wheelchair to move freely without constriction and also avoided noise and interference problems which had been encountered with a wired link.

\subsection{Sensors}

Sensors are required to provide motion and force information for closed-loop control of the wheelchair, Fig. 2 shows various sensors mounted in the wheelchair, including three accelerometers; three gyros; four rotational encoders. Two of the accelerometers are placed adjacent to the front wheels to measure the side and driving force on the wheels. These front two accelerometers were mounted on aluminium brackets, designed to get the accelerometer chip itself in line with the drive wheel axes and as close to the centre of the wheel as possible. The third accelerometer was placed on the back support between the castor wheels to measure forces on the casters and helps determine wheelchair skid or over-steer.

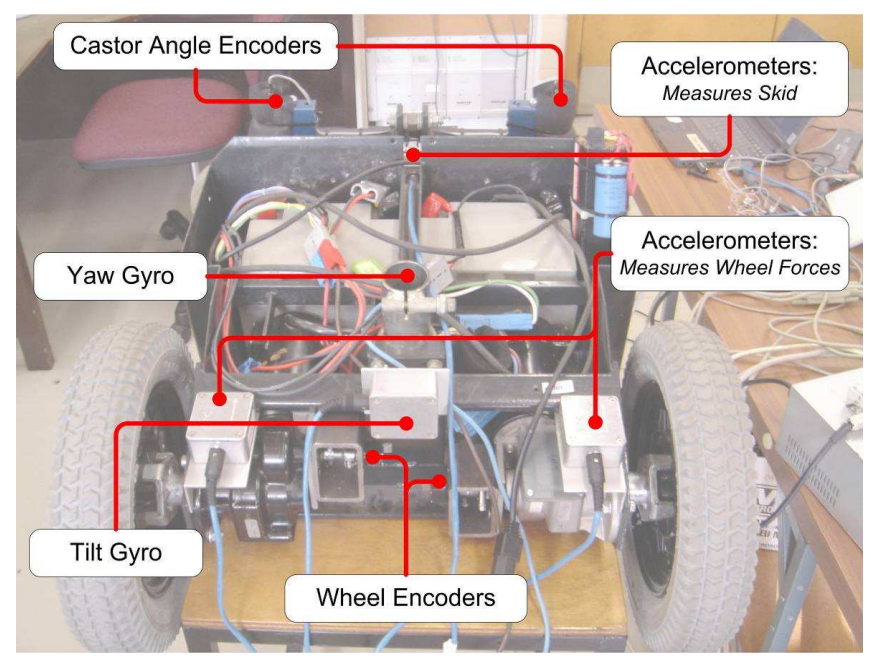

Fig. 2. Sensor placement.

Two of the gyros were placed to measure the tilt (sideways tip of the wheelchair) and yaw (turning rotation of the wheelchair about its vertical axis). For the gyro intended to measure tilt, a bracket was made, sitting in the centre between the drive wheels. It holds the gyro so that its rotational axis is in line with the direction of travel. The gyro to measure yaw can be set anywhere along the vertical axis passing through the Centre of Gravity (COG). The third gyro preferably is mounted exactly at the COG and is for future expansion and could be used to help determine jerk, or the degree to which the wheelchair rocks backwards and forwards.

The front two wheel encoders are for experimental purposes to give a relative $\mathrm{XY}$ position and path over the ground. The rear encoders on the castors are necessary when the model starts to include effect of the castor angle. Encoders were also installed for the front wheels. All encoders were mounted in custom built housings.

In addition to the wheelchair components itself, the wheelchair needs to also carry several items to provide wireless capabilities. These include sensor data acquisition box, the Bluetooth modules box, the Roboteq controller and $\mathrm{R} / \mathrm{C}$ receiver circuit. Housing was designed to house these items permanently, conceal the batteries and some of the wirings. 


\subsection{Motion Control}

The RoboteQ AX2550 motion controller was utilised to control the motors on the wheelchair. The AX2550 is capable of controlling two DC motors separately in forward and reverse directions. It is able to channel up to $120 \mathrm{~A}$ per motor. It has several control modes including: Serial RS23, Radio-Control PWM, and 0-5V Analog Voltage Input. Fig. 3 shows the RoboteQ motor controller mounted on the wheelchair.

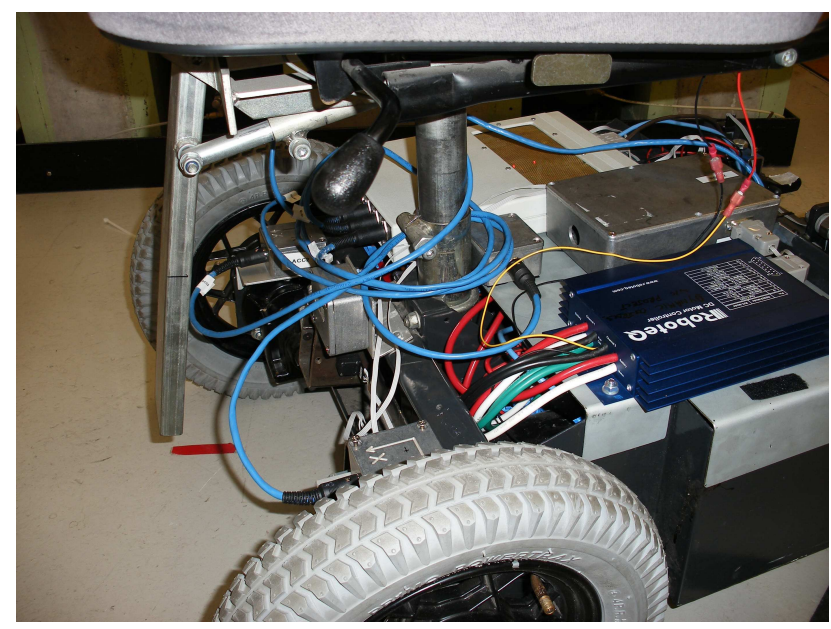

Fig. 3. RoboteQ Controller and battery connections.

The motor controller is set to receive Radio-Control PWM in the following manner

1. A duty cycle in the range of $\pm 100 \%$ is sent as unsigned 8 bit integers with a +100 offset in Real-Time from DSpace, via a RS232 Bluetooth device, for both the left and right motors.

2. The signal is received by a paired RS232 Bluetooth device and fed into the ' $\mathrm{R} / \mathrm{C}$ receiver circuit' which outputs the individual required PWM signals for the left and right motors (Fig. 4 ).

3. The PWM signals are a modulated $50 \mathrm{~Hz}$ signal where $5 \%$ duty cycle $=$ full reverse, $7.5 \%=$ stopped, $10 \%=$ full forward.

4. The $\mathrm{R} / \mathrm{C}$ receiver circuit contains a PSoC (Programmable System on Chip) device and a RS232 to TTL level converter (MAX232 Chip). The PSoC takes the input from the level converter via a UART receiver within the PSoC, performs several mathematical iterations to convert the values received from DSpace in step 1. to the required duty cycles (step 3.) which are then set in 2 separate PWM blocks, also contained within the PsoC. Fig. 5 shows the Simulink Model with demanded duty cycles from "PWM2.mat" file.

5. The separate PWM blocks output the demanded duty cycles for the left and right motors which are input directly to the RoboteQ motor controller.

For initial model validation, the low control frequency of 50 $\mathrm{Hz}$ is adequate to control the wheelchair wirelessly to obtain the necessary data, although the $20 \mathrm{~ms}$ cycle is far too slow for dynamic feedback control.

The flexibility of the current system, which uses a PSoC chip in the ' $\mathrm{R} / \mathrm{C}$ receiver circuit', allows for the chip to be reconfigured to output analogue signals via the same pins as are currently used for PWM output. This will allow for a much faster control loop rate than the current PWM and will be instituted at the feedback control phase of the project.

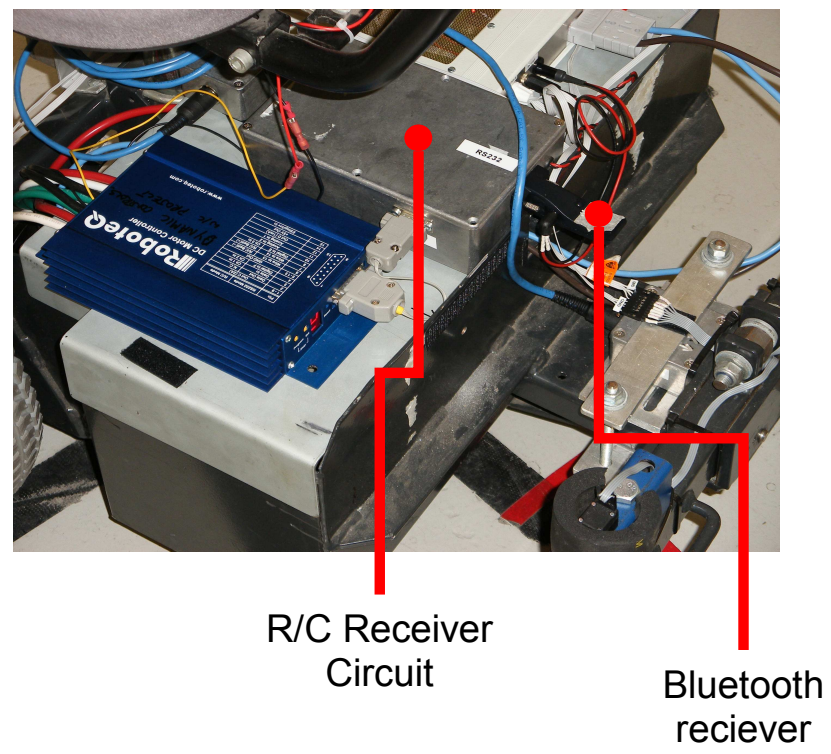

Fig. 4. Bluetooth device and $\mathrm{R} / \mathrm{C}$ receiver.

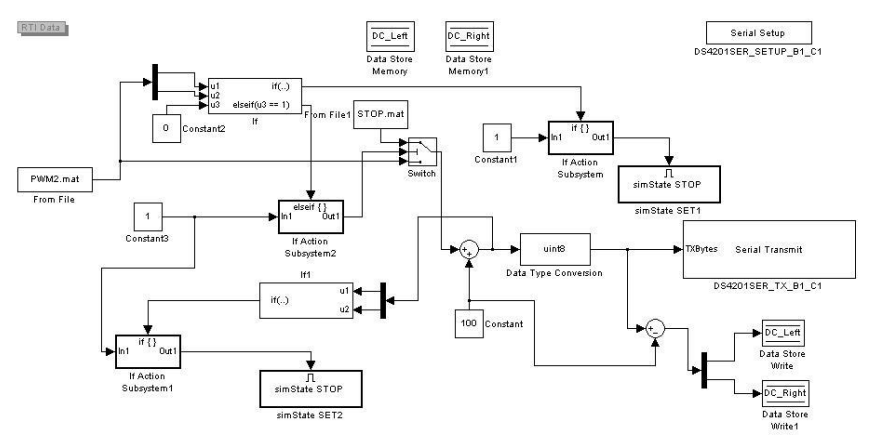

Fig. 5. Simulink model with demanded duty cycles.

\section{PARAMETERISATION OF WHEELCHAIR}

\subsection{Centre of Gravity (COG)}

Through the construction of a quadrifilar pendulum and the use of load cells in differing configurations, physical characteristics relating to the wheelchair such as the mass, centre of gravity and moment of inertia were determined.

Fig. 6 shows the individual equipment and interconnections. The load cell senses the weight of the wheelchair. Its output is conditioned by the amplifier. The amplifier output is read by the Digital Multi-Meter (DMM). 


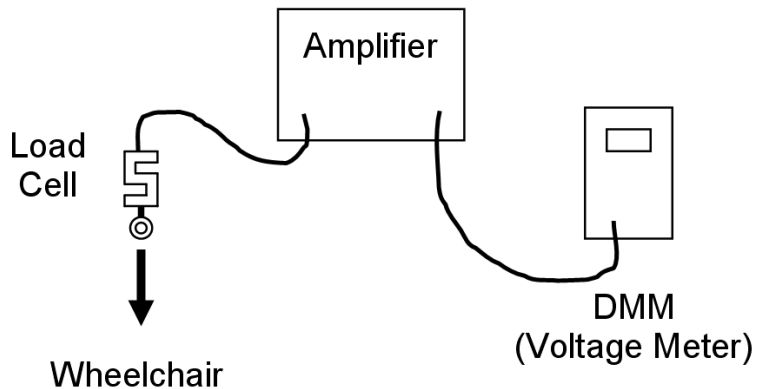

Fig. 6. Weight measuring equipment set-up.

The wheelchair was hung via steel wire cables to an eyehook connected to the load cell. The load cell was hung from a crane in the Mechanical Wing Workshop. The weights of the wheelchair can be calculated in the following formula.

$$
\begin{aligned}
\text { Weight } & =\frac{\text { Load cell weight limit } \times \text { DMM Voltage reading }}{\text { Amplifier gain } \times \text { Load cell supply voltage } \times \text { Load cell full scale output }} \\
& =\frac{1000 \mathrm{~kg} \times \text { DMM Voltage Reading } \mathrm{V}}{5800 \times 3.98 \mathrm{~V} \times 1.998 \mathrm{mV} / \mathrm{kg}} \mathrm{kg}
\end{aligned}
$$

The wheelchair centre of gravity (COG) was found using simple lever arm calculations. The experimental setup is shown in Fig. 7. The wheelchair was securely mounted on a platform. The overall weight was measured by a digital load cell. To determine the location of the COG, several load measurements were taken on each side. These, combined with the pivot and lifting point locations and total weight, allowed COG to be established.

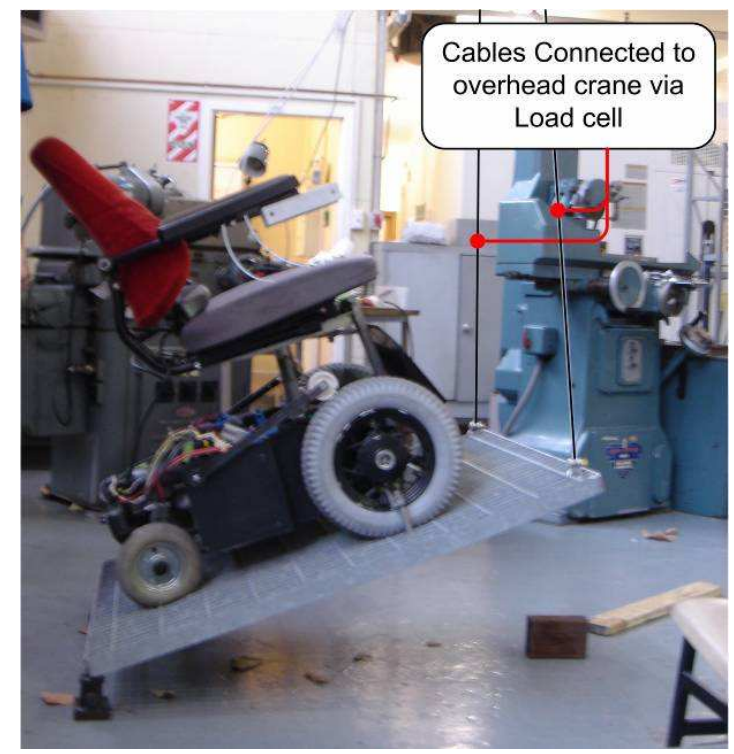

Fig. 7. Experimental setup to determine centre of gravity.

\subsection{Moments of Inertia}

A Quadrifilar pendulum was used to find the moments of inertia of the wheelchair, shown in Fig. 8. The mass moment of a quadrifilar pendulum about its vertical axis, was obtained from the theory:

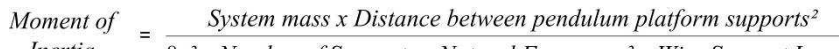

Inertia $=\overline{8 \pi^{2} x \text { Number of Supports } x \text { Natural Frequency }{ }^{2} x \text { Wire Support Length }}$

$$
I_{z}=\frac{m g D^{2}}{32 \pi^{2} f_{n}^{2} h} \quad \mathbf{k g} \cdot \mathbf{m}^{2}
$$

Before the moments of inertia for the wheelchair could be obtained the moment of inertia of the pendulum first needed to be calculated. From this, the correct moment of inertia for each of the wheelchair's axes could accurately be obtained by subtracting the pendulum's moment of inertia from the total inertia.

To find the natural frequency of the system, the wheelchair's centre of gravity was placed over the centre of the pendulum's circular platform. This was done for all three axes of the wheelchair.

The pendulum was then set in motion, by turning the platform approximately $5^{\circ}$, and the period of oscillation was measured. The natural frequency was obtained from this data, and used to calculate the inertia for that orientation.

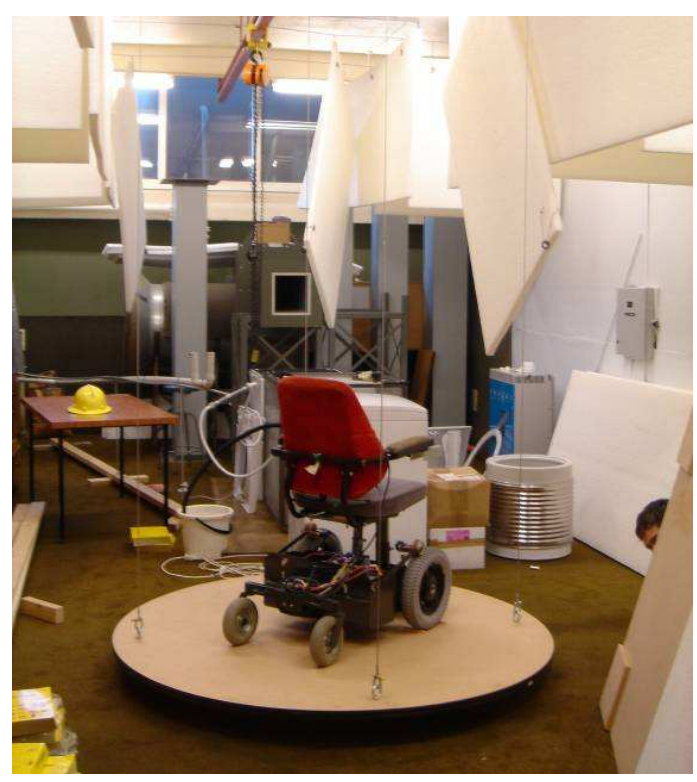

Fig. 8. Quadrifilar Pendulum setup for moment of inertia

\subsection{Motor Constants}

In order to accurately model the dynamics of the wheelchair, the inner workings of the motors had to be investigated. This involved research into how DC motors operated. Equations governing the motors inputs and outputs were ascertained, as shown below.

$$
\begin{aligned}
V & =L_{a} \frac{d i}{d t}+R_{a} i+K_{m} \dot{\theta} \\
\tau & =K_{t} i=J \ddot{\theta}+B \theta \\
& =F r=I \ddot{\theta}=m r^{2} \ddot{\theta}
\end{aligned}
$$

In State Space form, 
$\left[\begin{array}{c}\ddot{\theta} \\ \frac{d i}{d t}\end{array}\right]=\left[\begin{array}{cc}\frac{-B}{J} & \frac{K_{t}}{J} \\ \frac{-K_{m}}{L_{a}} & \frac{-R_{a}}{L_{a}}\end{array}\right]\left[\begin{array}{l}\dot{\theta} \\ i\end{array}\right]=\left[\begin{array}{c}0 \\ i \\ \frac{i}{L_{a}}\end{array}\right] V$

$\dot{\theta}=\left[\begin{array}{ll}1 & 0\end{array}\right]\left[\begin{array}{l}\dot{\theta} \\ i\end{array}\right]$

where $\mathrm{i}=$ current; $\mathrm{L}_{\mathrm{a}}=$ motor inductance, $\mathrm{R}_{\mathrm{a}}=$ motor resistance, $\mathrm{V}=$ voltage, $\mathrm{K}_{\mathrm{m}}=$ motor speed constant, $\operatorname{dot}(\theta)=$ Angular speed of motor, $\mathrm{J}=$ inertia of motor and wheel, $\mathrm{B}=$ Coulomb friction of motor and load, $\mathrm{K}_{\mathrm{t}}=$ motor torque constant, $\tau=$ torque supplied by motor, $\mathrm{F}=$ force on wheels, $\mathrm{r}=$ radius of wheels.

The constants contained in the equations were determined experimentally. These values could then be fed straight into the model for a more accurate representation of the wheelchair. The measurement data of the speed against the voltage are shown in Fig. 9. The speed constant $\mathrm{K}_{\mathrm{m}}$ of the DC motor is the slope of the plot of the speed versus the voltage. It is calculated to be $1.765 \mathrm{rad} / \mathrm{s} /$ volt. Likewise experimentation was carried out to plot the torque generated versus the armature current, the torque constant of the DC motor, $\mathrm{K}_{\mathrm{t}}$, was calculated to be $1.739 \mathrm{Nm} / \mathrm{A}$.

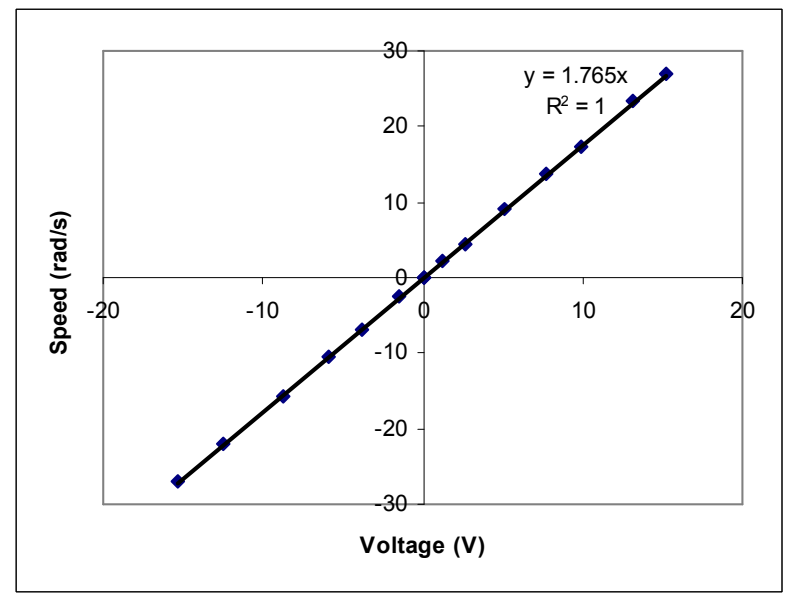

Fig. 9. Motor speed against voltage.

\section{Wheelchair Model}

The mathematical model of the wheelchair takes as inputs the voltages to the two driving motors and outputs the position of the chair in the X-Y plane. The advantages of having an accurate software model of the wheelchair can not be understated, and once validated will be an invaluable tool for rapid prototyping of a control system.

The choice to use Simulink as the platform to develop the model was due to two reasons. Firstly, Simulink is the dedicated platform of choice for interface with dSPACE through ControlDesk. Secondly, and most importantly, the equations derived could not be put in State Space form, as initially desired, due to non-linear sinusoid coefficients. State Space form would have enabled the use of simpler Matlab Mfiles and more advanced feedback control options.
Two separate basic models were created. The first model, a purely kinematics model, treats the wheelchair as two wheels separated at a distance. It is governed by the following equations:

$\dot{\theta}=\frac{r}{d_{2}}\left(\omega_{1}-\omega_{2}\right)$

$v_{u}=\frac{\left(\omega_{1}+\omega_{2}\right) r}{2}$

$v_{w}=\dot{\theta} d$

$\dot{\theta}$ is the yaw rate, $v_{u}$ and $v_{w}$ the forward and sideways speeds of the wheelchair respectively, and $\omega_{1,2}$ the rotating speeds of the front wheels.

The above kinematics model proved to be robust and seemingly accurate. But it was not expandable to meet the requirements of the control prototyping application.

The second prototype model treats the wheelchair as a two dimensional object, and focuses on the forces and moments acting on the chair. It proved difficult to get the model to follow an expected path. However its ability to be adjusted made it the preferred choice for developing the control prototyping system further. Another advantage of the force model was that the data available from the accelerometers and gyros, in particular the forces on the wheels, could be compared directly to parts of the model.

The model was initially based around a simple free body diagram involving two dimensional forces on the driving wheels and the centre of gravity, which was assumed to be in the middle of all four wheels. Over time, additions were made such that the final free body diagram evolved into that pictured below. The model was further enhanced to include forces on both driving wheels and passive wheels, and to allow for the offset of COG from the geometric centre of four wheels, as illustrated in Fig. 10.

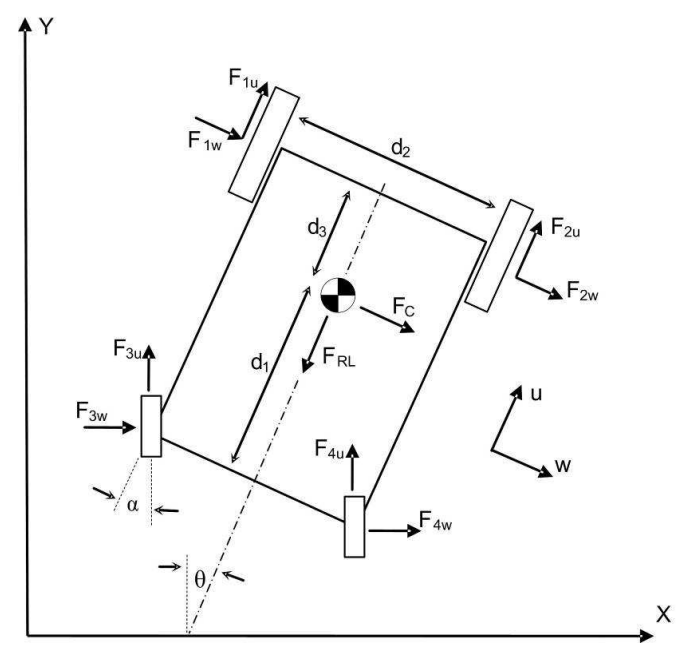

Fig. 10. Free body diagram of wheelchair. 
By summing all forces in both the local $\mathrm{u}$ and $\mathrm{w}$ directions, as well as taking moments about the centre of gravity, both rotational and translational accelerations are determined.

The driving forces on the two front wheels, $F_{1 u}$ and $F_{2 u}$, were calculated using the DC motor equations. These equations relate the voltage across the motors to the output torque on the wheels. Knowing the torque, $\tau$, the linear force, $F$, can easily determined as follows:

$$
F=\frac{\tau}{r}=\frac{I \alpha}{r}=\frac{I \ddot{\theta}}{r}
$$

Modelling the side forces on the wheels was not as straight forward as expected. When travelling in a straight line, these forces equal zero and when cornering they are related to how sharp and fast the turn is. For modelling purposes, the centripetal force acting through the centre of gravity was split into four forces acting on each wheel depending on their distance to the centre. This centripetal force is directly proportional to the yaw rate, $\dot{\theta}$, and the forward speed of the chair, $v_{u}$.

$F_{c}=\frac{m v_{u}^{2}}{r}=m v_{u} \dot{\theta}$

where $m$ is mass.

The Sum of lateral forces on chair equals the centripetal force on chair:

$\sum F_{\text {lateral }}=F_{c}$

$\therefore F_{\text {lateral,rear }}+F_{\text {lateral, front }}=\frac{m v_{u}^{2}}{r}=m v_{u} \dot{\theta}$

Also, moments from these side forces must equal zero about C.O.G:

$$
F_{\text {lateral,rear }} d_{1}-F_{\text {lateral,front }} d_{3}=0
$$

Combining above equations gives expressions for the lateral forces on the chair:

$$
\begin{aligned}
& F_{1}=F_{2}=\frac{d_{1}}{2\left(d_{1}+d_{3}\right)} m v_{u} \dot{\theta} \\
& F_{3}=F_{4}=\frac{d_{3}}{2\left(d_{1}+d_{3}\right)} m v_{u} \dot{\theta}
\end{aligned}
$$

Bluetooth and Radio-Control receiver, has been has been employed to control the two DC motors of the wheelchairs. Test procedures have been designed to find parameters of the wheelchairs, including centre of gravity and moment of inertia. Motor constants including speed constant and torque constant can be derived from the experimental study. Both kinematics and force model of the wheelchair were established, using Simulink as a platform. The established model will be validated in the follow-up work.

\section{REFERENCES}

Boiadzjiev, G., D. Stefanov (2002). Powered wheelchair control based on the dynamical criteria of stability", In: Mechatronics, Vol. 12, No. 4, May 2002, pp. 543-562.

Boquete1, L., R. García1, R. Barea1 and M. Mazo (1999). Neural Control of the Movements of a Wheelchair. In: Journal of Intelligent and Robotic Systems, Vol. 25, No. 3, pp. 213-226.

Bureau of the Census (1999). World Population at a Glance: 1998 and Beyond. U.S. Department of Commerce, Economics and Statistics Administration, BUREAU OF THE CENSUS, IB/98-4, Issued January 1999.

Ding, D., R.A. Cooper, S. Guo, T.A. Corfman (2004). Analysis of Driving Backward in an Electric-Powered Wheelchair. In: IEEE Transactions on Control System Technology, Vol. 12, No. 6, pp. 934-943.

Frost \& Sullivan (2004). North American Mobility Aids Markets. October 7, 2004, Pub ID: MC1052625.

Kamper, D., D.M. Parnianpour, K. Barin, T. Adams, M. Linden, H. Hemami (1999). Postural stability of wheelchair users exposed to sustained, external perturbations. In: Journal of Rehabilitation Research \& Development, Vol. 36 No. 2.

Linden, M., D. Kamper, S. Reger, T. Adams (1996). Transportation needs: survey of individuals with disabilities. In: Proceedings of the 19th Annual RESNA Conference; 1996 June 7-12, Salt Lake City, UT. Washington, DC: RESNA Press, pp. 52-54.

NHS (1966). 1996 National Prosthetic \& Wheelchair Services Report (1993-1996), UK.

WinterGreen Research Inc. (2004). Wheelchair Market Opportunities, Strategies, and Forecasts, 2004 to 2009 , October 2004.

Stefanov, D.H., Z. Bien, W.K. Song, J.S. HAN (2000). Some aspects of human-friendly control for movement-helping devices. In: Journal of Artificial Life and Robotics. Vol.4, No. 4, December 2000, pp. 198-205.

\section{CONCLUSIONS}

A wireless data acquisition and control platform integrates sensors, motion controller, and the motorised mechanical system. The RoboteQ motion controller, with RS232 